\title{
Metodología de la Bioética. Análisis de casos clínicos
}

\author{
Dra. Mg. María Teresa Rotondo de Cassinelli \\ Palabras clave: \\ BIOÉTICA CLÍNICA \\ BIOÉTICA SOCIAL \\ Key words: \\ CLINICAL BIOETHICS \\ SOCIAL BIOETHICS
}

\section{Introducción}

La importancia de un método o procedimiento en la toma de decisiones se debe a los beneficios que brinda, especialmente en situaciones conflictivas, orientando el análisis de los dilemas éticos que surgen en el ámbito de la atención de la salud. En las disciplinas formales y abstractas, como la lógica y las matemáticas, pueden alcanzarse certezas; en cambio, en otras, como la medicina, en las que se deben tener en cuenta circunstancias y consecuencias del accionar, no caben más que incertidumbres y probabilidades.

Gracia $^{(1)}$ cita a Aristóteles en su afirmación de que el saber sobre lo particular no puede aspirar más que al estatuto de lo probable. Y se pregunta cómo tomar decisiones racionales en el ámbito de la clínica médica, donde carecemos de certeza y tenemos un alto porcentaje de incertidumbre. Sostiene que no solo en el terreno de la técnica, sino también en ética, es necesario el uso de métodos, siendo mayor la necesidad cuanto más ambiguas sean las experiencias o cuando generen más incertidumbres.

\section{Procedimientos de decisión (segunda parte)}

Seguiremos con la presentación de los procedimientos de decisión usados en ética clínica, comenzada en el número anterior, en el que se expuso el enfoque principalista.

\section{B. Enfoque casuístico}

Jonsen, Siegler y Winslade(2) presentan una perspectiva inscrita en la renovación de la antigua casuística; consideran que en ética el procedimiento de análisis no debe partir de los principios, sino de las situaciones particulares, y que es posible considerar los casos estudiando inicialmente las circunstancias concretas de cada uno. Este método alude a la casuística clásica, que era una forma de razonar a partir de "lugares comunes" o "topos". Estos "topos" o tópicos servían para encuadrar los casos; posteriormente, Cicerón amplió los términos y planteó ¿quién lo hizo?, ¿en qué circunstancias?, ¿y con qué fin?

En Bioética se usa este método cuando no hay acuerdo en los fundamentos o principios morales y se procede a encontrar el caso paradigmático (por ejemplo, Karen Quinlan, Baby Doe, etcétera). Esta forma de reflexionar, que compara los casos en estudio con los paradigmáticos es útil, dado que los casos previos ya han sido puestos a prueba y se discutieron y difundieron los argumentos planteados en esas ocasiones. Así se analizan las máximas o normas morales que se han puesto en juego y se estudian las circunstancias del caso y sus diferencias con el caso paradigmático. Jonsen, Siegler y Winslade no plantean principios, sino que proponen tener en cuenta las máximas derivadas de deliberaciones prudentes. Presentan el siguiente orden para el análisis:

1) Preferencias del paciente. 2) Indicaciones médicas. 3) Calidad de vida. 4) Factores socioeconómicos.

En relación con las máximas, Brussino expresa: "A diferencia de los principios, que son a priori, las máximas son el resultado de un procedimiento inductivo por el cual, partiendo de casos similares se generaliza una norma moral, probablemente aplicable a futuros casos similares"(3).

\section{Enfoque del contextualismo}

En este procedimiento, las acciones humanas co- 
bran sentido en la interpretación de sus elementos contextuales y no en el seguimiento de normas y reglas morales.

El contexto incluye consideraciones sobre el carácter, los sentimientos, las narrativas de vida, así como sobre la cultura y tradición del medio de donde provienen los implicados en el caso. Entre las éticas que ponen en consideración estos elementos se encuentra la teoría narrativa.

\section{Enfoque basado en las éticas de la virtud}

A diferencia del método casuístico que estudia los actos morales, este método se enfoca más en el carácter moral de los agentes. Para Drane, la ética no solo trata de acciones, sino también de hábitos (virtudes) y de actitudes (carácter) ${ }^{(4)}$.

Por su parte, Beauchamp y McCullough establecen: "Dichos hábitos o rasgos de carácter tienen una importancia radical en el apresurado contexto de la práctica médica. El objetivo de cultivar las virtudes es hacer del cumplimiento de deberes una cuestión de comportamiento habitual, más que una lucha constante para poner en práctica lo que demanden los principios morales". "En la teoría ética, las virtudes morales son disposiciones o hábitos firmemente asentados que están casi siempre correlacionados con principios morales de deber. El lenguaje de las virtudes refuerza más que suplanta al de los principios de deber, ya que tanto los deberes como las virtudes son necesarios para que la responsabilidad moral sea un 'contrapeso al egoísmo' y así dirija al médico hacia los mejores intereses del paciente"(5). Sostienen: "El médico debe apoyarse en virtudes como: respeto, honestidad, tacto, discreción, compasión, simpatía y tolerancia. Estas virtudes avivan y nutren lo humano de la medicina, y a veces transforman difíciles problemas de conflicto en intercambios dignos y humanizados"(5).

\section{E. Otros enfoques}

Corresponden a las corrientes siguientes: Hermenéutica, Ética del cuidado y Derechos Humanos. Actualmente existe un creciente interés sobre el enfoque de salud y derechos humanos. En 2005 se aprobó en Francia la Declaración Universal sobre Bioética y Derechos Humanos ${ }^{(6)}$. Sobre este documento, Garrafa opina que "es el documento público más importante producido colectivamente sobre la Bioética en este siglo XXI. Su contenido transformó la agenda bioética internacional, (re)fundando su epistemología y expandiendo sus fronteras de actuación. La inclusión de las temáticas sanitarias, sociales y ambientales en el cuerpo del documento, más allá del contenido exclusivamente biomédico-biotecnológico, proporcionó una verdadera poli- tización de la ética aplicada al campo sanitario"(7). La Declaración Universal sobre Bioética y Derechos Humanos hace especial referencia, en su artículo 14, a la responsabilidad social y a la salud.

Existen múltiples tipos de procedimientos de decisión ${ }^{(1)}$; destacamos el método presentado por el director del Centro de Bioética de la Universidad de Bochum (Alemania) y del Programa Europeo del Kennedy Institute de Washington, Hans Martin Saas, que ha sido publicado en 1987 y usado frecuentemente en Latinoamérica (tabla 1) ${ }^{(8)}$.

"La asignación de recursos a la asistencia sanitaria no solo supone un tema de costo-beneficio-eficacia, sino que, por encima de todo, es un tema de justicia humana o equidad en el que es prioridad sopesar debidamente los intereses de cada una de las partes implicadas"

En los países europeos, de tradición humanista, el uso del método principalista se ha complementado con el resurgimiento del interés sobre las virtudes morales. Esto lo diferencia del primer modelo norteamericano, en el cual hay un neto predominio del principio de autonomía vinculado al marcado individualismo reinante en dicha sociedad. En Latinoamérica se acogió este modelo, imponiéndose como una novedad el auge de la autonomía y terminando con la implantación del consentimiento informado como una exigencia dentro de la relación sanitaria. Sin embargo, se observa que aunque se han tomado elementos provenientes de la influencia norteamericana, es fuerte aún la impronta humanística en nuestras regiones. Adela Cortina, refiriéndose a los posibles rasgos de una bioética latina, cita a Schtosmans, quien compara la bioética angloamericana con la europea. En el año 2001, Schtosmans presenta la síntesis de su estudio, que se ilustra en la tabla $2^{(9)}$. Actualmente los mayores conflictos o dilemas éticos originados en las nuevas realidades sociales, económicas y culturales de nuestras sociedades están vinculados a la interpretación y al ejercicio del principio de justicia. En los países de escasos recursos económicos, con poblaciones vulnerables y con menor desarrollo de las tecnologías, la asignación de recursos sanitarios, los criterios de racionalización y la naturaleza de las obligaciones de los Estados, son temas que producen gran preocupación. 
Tabla 1. Protocolo de Bochum para la práctica ético-médica. Análisis científico y ético para el manejo de un caso médico.

\section{Identificación de los hallazgos cientifico-médicos}

El relevamiento de hallazgos sigue principios reconocidos y aceptados para el diagnóstico científico:

Reflexiones generales

¿Cuál es el diagnóstico del paciente y cómo e su pronóstico?

¿Cuál tratamiento puede proponerse desde el punto de vista médico ante tal diagnóstico y pronóstico? ¿Cuáles alternativas terapéuticas pueden indicarse?

¿Cuál es la probabilidad de éxito previsible ante las posibilidades terapéuticas propuestas?

¿Cómo es el pronóstico sin los tratamientos propuestos?

Reflexiones especiales

¿Servirá al paciente el tratamiento contemplado médicamente?

¿Influirá este favorablemente al pronóstico general en el caso especial?

¿En qué grado?

¿Podría perjudicar al paciente en relación con su curación o bienestar?

¿En qué medida? ¿Cómo se sopesan beneficios y perjuicios unos contra otros? Obrar del médico

¿Existen condiciones adecuadas de tratamiento: personales, equipo, aparato técnico, consideración del estado de la investigación médica y de la experiencia del médico?

¿Qué factores importantes se desconocen?

¿Son los conceptos médicos clave suficientemente claros?

Resumen:

¿Qué tratamiento sería óptimo en vista del hallazgo científico-médico?
Identificación de los hallazgos ético-médicos

La base para el relevamiento de hallazgos es una valoración según los siguientes principios:

Salud y bienestar del paciente

¿Cuáles perjuicios pueden acarrear cada una

de las alternativas terapéuticas?

¿Empeoramiento del bienestar, dolores,

acortamiento de la vida?

¿Molestia física o espiritual del paciente?

Autodeterminación del paciente

¿Qué se sabe acerca del sistema de valores de paciente?

¿Qué actitud tiene el paciente frente a las

formas de tratamiento médico (intensivas, paliativas o de reanimación)?

¿Está el paciente suficientemente informado acerca del diagnóstico, pronóstico y terapéutica?

¿Hasta qué punto se puede tener en cuenta al paciente en la valoración, o hasta qué punto puede ser completamente dejado de lado?

¿Quién sino él puede tomar decisiones en su lugar?

¿Consiente el paciente a la terapia?

Responsabilidad médica

¿Existen conflictos entre los juicios éticos del médico, del paciente, del equipo asistencial o de la familia?

¿Puede solucionarse o suavizarse tal conflicto mediante una determinada opción

terapéutica?

¿Cómo se asegurará que, en especial, no se

lesionarán los siguientes principios: la relación de confianza entre el médico y el paciente, el principio de veracidad, y el secreto

profesional?

¿Qué factores importantes se desconocen? ¿Son suficientemente claros los conceptos éticos clave utilizados y la relación entre ellos?

Resumen:

¿Cuál tratamiento sería el óptimo en vista del hallazgo ético-médico?
Manejo del caso

¿Cuáles opciones (alternativas) de solución se ofrecen en vista de un posible conflicto entre los hallazgos científico-médicos y los ético-médicos?

¿Cuáles de los antedichos criterios científicos y éticos se ven afectados por estas opciones?

¿Cuáles opciones serían las más adecuadas al perfil de valores del paciente?

¿Quién podría o debería ser convocado como consejero?

¿Está indicada la transferencia del caso a otros por razones médicas o éticas? ¿Cuáles son las obligaciones concretas del médico para el tratamiento elegido?

¿Cuáles son las obligaciones del paciente, del personal auxiliar, de la familia, de las organizaciones para la salud pública?

¿Hay argumentos contra la decisión? ¿Cómo confrontar esos argumentos? ¿Está la decisión éticamente consentida?

¿Por quién?

¿Por qué?

¿Sería ella discutida con el paciente y alcanzado su consentimiento?

¿Revisión de la decisión?

Resumen:

¿Cuál decisión se tomó en vista del conjunto de hallazgos científico-médicos y ético-médicos, y ante la ponderación de valores realizada? ¿Cómo se pueden resumir breve y claramente la decisión ético-médica y la ponderación de valores realizada?

Preguntas adicionales para la valoración ética

1. En casos de tratamiento prolongado: ¿Se revisa rutinariamente el tratamiento médico seguido y su valoración ética? ¿Es el tratamiento suficientemente flexible para adecuarse a los cambiantes hallazgos científico-médicos?

¿Qué va a cambiar en el curso del tratamiento por la imprevista presentación de hallazgos nuevos científico-médicos y ético-médicos? ¿Cómo se halla el paciente ante cambios de estrategia de tratamiento?

¿Sigue, a más infausto pronóstico, una ponderación entre medidas terapéuticas paliativas o médico-intensivas?

¿Está asegurado que con esto se considera la voluntad expresa o presunta del paciente?
2. En caso de acentuada relevancia social: ¿Qué complicaciones familiares, emocionales, profesionales o económicas, relevantes para el estilo de vida, se originan?

¿Pueden estas complicaciones ser sobrellevadas por el paciente, por sus parientes o por la comunidad?

¿Se promueve la integración social del paciente, su alegría de vivir y el desarrollo de su personalidad?

¿Qué significado tiene la respuesta a estas preguntas para la ponderación de valores científico-médicos y ético-médicos?
3. En casos de investigación terapéutica o no terapéutica:

¿Es óptima la disposición de la prueba experimental en vista de los aspectos ético-médicos?

¿Es la investigación necesaria?

¿Ha dado el voluntario sano o el paciente su consentimiento después de ser informado?

¿Por cuáles motivos podría la información ser incompleta o no ser comprendida enteramente? ¿Qué motivos podrían darse para que el voluntario sano o el paciente no haya prestado su consentimiento de manera absolutamente voluntaria?

¿Se ha asegurado que en la elección de los pacientes, estos no han sido favorecidos o perjudicados respecto a otros pacientes? ¿Tiene el voluntario sano o el paciente en todo momento el derecho de renunciar a su participación en la investigación, y se le comunicó esto de manera suficiente y comprensiva? 


\begin{tabular}{|ll}
\hline Tabla 2. \\
\hline Bioética angloamericana & Bioética europea \\
\hline Principalismo & Tradición hipocrática \\
Sistema sanitario privado & Sistema sanitario socializado \\
Base de asistencia: derechos & Base de asistencia: solidaridad \\
Principio de autonomía & Prioridad de la relación médico-paciente \\
Conversión en bioderecho & Relevancia del ethos \\
Utilitarismo - Pragmatismo & Tradiciones continentales \\
\hline $\begin{array}{l}\text { Schotsmans P. La bioética en Europa. Citado por Cortina A. en: Tareas de una bioética latina. Jurisprudencia Argentina, } 2004 \\
(5): 9-15\end{array}$
\end{tabular}

\section{Análisis ético de dos casos clínicos}

Como ejemplo de uso de procedimientos, realizaremos el análisis de dos casos clínicos publicados en el número anterior aplicando diferentes métodos.

\section{Análisis del caso $1^{(10)}$}

Para su estudio seguiremos el Protocolo de Bochum de Hans-Martin Saas, el que se resume en tres pasos principales:

\section{1) Identificación de los hallazgos científico-médicos}

El análisis de los datos clínicos siempre es de resorte científico-médico (de los especialistas tratantes, poseedores de conocimientos actualizados). Cuando un caso se plantea ante un comité de ética clínica, las respuestas a las interrogantes han de ser aportadas por los médicos tratantes que presentan el caso, probablemente ya discutido en el ámbito científico correspondiente. Por lo tanto, no nos corresponde adentrarnos más en este tema.

\section{2) Identificación de los hallazgos ético-médicos}

En este segundo paso se trata de valorar la salud y el bienestar del paciente, su autodeterminación y la responsabilidad médica. De la historia presentada podemos deducir que ha habido una ponderación de todas las interrogantes y que el paciente siguió la indicación de someterse al tratamiento financiado por el Fondo Nacional de Recursos (FNR). No surge que haya existido conflicto entre los juicios médicos, ni entre las decisiones del paciente y su familia.

\section{3) Manejo del caso}

De las preguntas que el protocolo presenta en este paso, destacamos las siguientes:

Caso 1

GBM, sexo masculino, 55 años (fecha nacimiento 2/7/1961). AP: dislipemia, sobrepeso, exfumador.

Infarto de región anterior en 2001 y de región inferior en 2017, revascularizado por angioplastia de descendente anterior y coronaria derecha (último procedimiento en febrero de 2017).

Motivo de consulta: síncope; enviado a nuestro servicio $(24 / 8 / 2017)$.

EA: insuficiencia cardíaca clase funcional II. Sin ángor. ECG: ritmo sinusal con bloqueo completo de rama izquierda (QRS $180 \mathrm{~ms}$ ). Ecocardiograma: FEVI 30\%; ventrículo izquierdo dilatado con trastornos segmentarios de la contractilidad. Función renal normal (azoemia: 0,48 $\mathrm{g} / \mathrm{l}$; creatininemia $1,12 \mathrm{mg} / \mathrm{dl}$ ).

Tratamiento: carvedilol, valsartán, furosemide, espironolactona, atorvastatina, clopidogrel y AAS.

Considerando: síncope, miocardiopatía dilatada isquémica, sin comorbilidades, recibiendo tratamiento médico óptimo, con expectativa de vida mayor a un año y FEVI 30\% más bloqueo de rama izquierda, se envía al FNR solicitud de autorización para implante de DAI con terapia de resincronización con fecha 17/5/2017.

El 30/5/2017 recibimos respuesta del FNR solicitan-

do la realización de un Holter y de un estudio electrofisiológico (EEF).

Holter: ritmo sinusal con 500 extrasístoles ventriculares en 24 horas.

EEF: intervalo HV normal, no se inducen arritmias sostenidas.

Reconsiderado el caso por el Ateneo Médico del FNR se nos informa resolución de fecha 27/6/17: “...la solicitud se encuentra fuera de la normativa vigente del FNR en paciente que el EEF no induce arritmias ventriculares sostenidas".

Se plantea al paciente la realización de un recurso de amparo. Luego de considerar esta propuesta, el paciente y su familia deciden proceder con el proceso judicial.

La audiencia tiene lugar el día 21/7/17 (viernes). Al día siguiente el paciente tiene un episodio de muerte súbita cardíaca, es resucitado pero fallece 48 horas después.

Tres días después (25/7/17) somos informados de la resolución judicial que hace lugar al recurso presentado; el paciente ya había fallecido. 
Caso 2

MWZP, sexo masculino, 46 años (fecha nacimiento $16 / 12 / 1970$ ).

AF: hermano con miocardiopatía dilatada que fallece súbitamente a los 40 años.

AP: hipertensión, dislipemia, sobrepeso.

EA: enviado a nuestro servicio (24/8/2017) para valorar implante de DAI.

Insuficiencia cardíaca clase funcional II. ECG: ritmo sinusal con bloqueo completo de rama izquierda (QRS $160 \mathrm{~ms})$. Ecocardiograma: FEVI 22\%; ventrículo izquierdo dilatado sin trastornos segmentarios de la contractilidad. Centellograma de perfusión con isonitrilo sensibilizado con dipiridamol: sin evidencias de isquemia. Función renal normal (azoemia: 0,33 g/l; creatininemia $0,72 \mathrm{mg} / \mathrm{dl}$ ).

Tratamiento: bisoprolol, enalapril, atorvastatina.

Considerando: paciente joven, con antecedente familiar de muerte súbita, portador de una miocardiopatía dilatada no isquémica con FEVI baja, sin comorbilidades, recibiendo tratamiento médico óptimo, con expectativa de vida mayor a un año y FEVI significativamente disminuida más bloqueo de rama izquierda, se envía al FNR solicitud de autorización para implante de DAI con terapia de resincronización con fecha 30/8/2017.

El 12/9/2017 recibimos respuesta del FNR: “...la solicitud se encuentra fuera de la normativa vigente para la financiación solicitada dado que el paciente no presenta arritmias de significación ni síncope. Se considerará la solicitud de implante de MP con resincronizador".

El paciente no acepta recibir solo un marcapaso ni desea utilizar el mecanismo del recurso de amparo.

Fallece súbitamente en la mañana del 1 de febrero de 2018 .

a. ¿Cuáles son las obligaciones concretas del médico para el tratamiento elegido?

Las obligaciones del médico hacia su paciente se resumen en su estudio y diagnóstico, informar al paciente de la naturaleza de su afección e indicarle el tratamiento y realizar las derivaciones correspondientes al no poder realizar el mismo en la institución sanitaria. El equipo médico cumple con orientar hacia otra vía para suministrar el procedimiento y así proteger los derechos del paciente.

b. ¿Cuáles son las obligaciones del paciente, del personal auxiliar, de la familia y de las organizaciones para la salud pública?

Debido a su elevado costo, el tratamiento no puede ser asumido por la institución sanitaria ni por su familia, por lo que se recurre al FNR que resuelve: "Reconsiderado el caso por el Ateneo Médico del FNR de resolución de fecha 27/6/17: “...la solicitud se encuentra fuera de la normativa vigente del FNR en paciente que el EEF no induce arritmias ventriculares sostenidas".

Se plantea al paciente solicitar el recurso de am- paro. "Luego de considerar esta propuesta, el paciente y su familia deciden proceder con el proceso judicial. La audiencia tiene lugar el día 21/7/17 (viernes). Al día siguiente el paciente tiene un episodio de muerte súbita cardíaca, es resucitado pero fallece 48 horas después. Tres días después (25/7/17) somos informados de la resolución judicial que hace lugar al recurso presentado; el paciente ya había fallecido".

El problema principal de este caso se debe a la carencia de recursos y a la forma en que éstos se asignan. Frente a esta situación se debería analizar la calidad de las decisiones en cuanto al uso racional de los recursos públicos disponibles. Debe tenerse cabal conocimiento de los criterios usados, si están basados en criterios científicos actualizados, transparentes e imparciales y, finalmente, asegurarse de la total ausencia de discriminaciones y de conflictos de intereses.

En resumen: el problema que dominó este caso fue la imposibilidad de llevar a cabo el correcto tratamiento por falta de recursos, teniendo como grave consecuencia la pérdida de una vida.

La aplicación justa de la distribución de recursos escasos es pues el problema más importante.

\section{B. Análisis del caso $2^{(10)}$}

Este análisis se realizará a través de otro procedimiento diferente del camino anterior (la palabra "méthodo" proviene del griego: poner en marcha, en camino). Se usará el método de los "principios morales", llamado también "principalismo", propiciado por Beauchamps y Childress ${ }^{(11)}$. Se le seguirá en el sentido inductivo, iniciando el examen desde los casos, para luego pasar por las reglas o normas morales.

\section{Nivel de los casos}

Corresponde a la presentación de los hechos científicos además de los sociales y económicos, de los deseos y valores de los pacientes.

De modo que en este nivel nos basamos en el resumen expuesto en este caso: "Considerando: $p a-$ ciente joven, con antecedente familiar de muerte súbita, portador de una miocardiopatía dilatada no isquémica con FEVI baja, sin comorbilidades, recibiendo tratamiento médico óptimo, con expectativa de vida mayor a un año y FEVI significativamente disminuida más bloqueo de rama izquierda, se envía al FNR solicitud de autorización para implante de DAI con terapia de resincronización con fecha 30/8/2017. El 12/9/2017 recibimos respuesta del FNR: “...la solicitud se encuentra fuera de la normativa vigente para la financiación solicitada dado que el paciente no presenta arritmias de significa- 
ción ni síncope. e considerará la solicitud de implante de MP con resincronizador". El paciente no acepta recibir solo un marcapaso ni desea utilizar el mecanismo del recurso de amparo. Fallece súbitamente en la mañana del 1 de febrero de 2018”.

\section{Nivel de las reglas o normas morales}

a) Veracidad. Se comunicó al paciente su diagnóstico, la naturaleza de su afección, la necesidad de un tratamiento y que el mismo dependía de otra institución. En la relación equipo de salud-paciente existió respeto por la regla o norma de veracidad al hacerle conocer la verdad de la situación.

b) Fidelidad. En la relación profesionales de la salud-paciente, con la promesa de respetar la relación fiduciaria, se ha priorizado el orientar a este último a tratar de conseguir lo mejor para él mismo, a pesar de conocer los inconvenientes $u$ otros intereses que se encontrarían para el logro del tratamiento.

c) Confidencialidad, privacidad. Evidenciamos el respeto en la publicación del caso al no figurar detalles personales (reserva de datos sensibles).

d) Consentimiento informado. Se desprende que se ha informado al paciente y se ha obtenido su consentimiento (sea escrito u oral) para los estudios, tratamientos y vías posibles.

En la última etapa el paciente tiene la opción de defender sus derechos por la vía judicial.

\section{Nivel de los principios}

a. No maleficencia. En relación con este principio, es con buena práctica médica, con indicaciones precisas, con competencia técnica, conocimientos actualizados y buenas relaciones interpersonales, que la relación sanitaria se cumple siguiendo el aforismo hipocrático: "Primum non nocere". (La evaluación de este nivel corresponde al ámbito científico, similar a lo visto en el primer paso del caso anterior: hechos científico-médicos).

b. Beneficencia. Se trató de procurar el bien del paciente, considerando la decisión de este y su preferencia.

c. Autonomía. Ha sido el propio paciente quien decidió seguir lo aconsejado en primera instancia y, por el contrario, no seguir el consejo en la segunda.

d. Justicia. La negativa de proporcionar el tratamiento desde la institución pública nos plantea la interrogante en cuanto a lo ético de la resolución.

En resumen: este segundo caso muestra cómo se respetaron los principios de no maleficencia, beneficencia, autonomía y cómo el conflicto se origina entre estos y el cumplimiento del principio de justicia.

\section{Comentarios}

Analizadas estas historias surgen variadas preguntas que toda la sociedad debe hacerse. Entre ellas: ¿Se ha respetado el principio moral de justicia? ¿De quién depende su cumplimiento? ¿Ha sido equitativo este final? ¿Existió igualdad de oportunidades? ¿Si hubieran obtenido recursos económicos a tiempo o apoyo económico familiar el final hubiera sido diferente? ¿ Se cumplió el ofrecimiento oportuno al acceso de los recursos? ¿Hubo discriminación?

Estas situaciones producen dudas, sufrimientos y angustias en enfermos, equipos médicos y entorno familiar y profesional. La sociedad entera se inquieta y entiende que existe una responsabilidad colectiva para evitar futuras e innecesarias muertes así como secuelas y sufrimientos.

En suma, en ambos casos estamos frente a:

- Un conflicto entre los principios morales (autonomía, no maleficencia, beneficencia versus justicia).

- Un dilema entre los intereses del bien privado y del bien común. El principal dilema ético que estos casos clínicos presentan es sobre el principio de justicia y da lugar a la deliberación sobre cómo y quién lo debe respetar. Como se había expresado anteriormente, la interpretación del principio de justicia está basada en el sistema de valores de la sociedad, que cristaliza finalmente en un marco legal adecuado.

En general se acepta que el principio exige equidad y no discriminación en la asignación de los recursos, así como sostenibilidad y acceso oportuno.

Hemos detectado un conflicto entre principios que tienen alcances diferentes y actores diferentes; el profesional no quiere actuar en forma maleficiente, sea ocultando o no informando -sobre el medicamento que el paciente debería recibir o sobre los procedimientos necesarios-aun conociendo las dificultades de su obtención. El paciente ve limitada su autonomía al no poder acceder al tratamiento por problemas económicos. El Estado se enfrenta con otras obligaciones, entre las que figuran lograr equidad en el reparto de los bienes finitos que posee y considerar accesibilidad, oportunidad y sustentabilidad de los mismos para toda la población.

Dado que el principal dilema ético que presentan estos casos trata del cumplimiento del principio de justicia, nos extenderemos sobre el tema de la justicia distributiva en el ámbito de la salud. 


\section{Justicia sanitaria}

La aplicación del principio de justicia en el ámbito de la salud, denominada justicia sanitaria, es un tema sobre el cual existen múltiples interpretaciones, tanto a lo largo del tiempo como en los diferentes lugares donde se ha aplicado. Sabemos que el cumplimiento de este principio que rige la vida de las sociedades depende de las políticas públicas de salud; que en su aplicación existen muchas y complejas dificultades por múltiples causas. Y resulta evidente que existen problemas para compatibilizar la equidad y la eficiencia en la gestión pública.

$\mathrm{Al}$ respecto, Beauchamp y Childress, refiriéndose a las teorías de la justicia (utilitaristas, liberta-

$\rightarrow$ "El debate de la asignación de recursos en salud ha sido dominado por un enfoque utilitarista o consecualista que determina el valor del acto médico y las políticas sanitarias exclusivamente en términos de consecuencias medibles o resultados" (Charlesworth)

rias, comunitaristas, igualitarias) expresan que solo esperan que "logren parcialmente dar coherencia y amplitud a nuestras visiones fragmentarias de la justicia social"(11), y agregan: "Buscamos proporcionar la mejor atención sanitaria para todos los ciudadanos, mientras que promovemos el interés público a través de programas de contención de costos. Promovemos el ideal del acceso igual a la asistencia sanitaria para todo el mundo, incluyendo el cuidado para los indigentes, mientras que mantenemos un ambiente competitivo de libre mercado. Estas metas deseables de asistencia superior, igualdad de acceso, libertad de elección y eficiencia social son difícilmente compatibles en un sistema social de libre mercado $^{(11)}$.

Según Lolas, "una distribución de recursos sanitarios realmente equitativa debe tener en cuenta tanto los valores, preferencias y expectativas de la sociedad como los principios teóricos de la justicia distributiva"(12). En este sentido, el concepto de equidad corresponde en general con el de un mínimo garantizado por el ideal igualitarista, aunque este planteamiento a menudo no se hace explícito, dados los eventuales costos políticos que puede acarrear.

Los criterios de selección de los servicios han de responder a problemas de equidad en la distribución de recursos y a la asignación racional entre grupos de distinto nivel de necesidad.

Se han de dar respuestas a situaciones del siguiente tipo: frente a la falta de recursos para dos aplicaciones, ¿debería reducirse la cobertura de las vacunaciones u otros programas esenciales para poder financiar el tratamiento de los pacientes con infección por VIH? ¿Sería preferible, si fuera posible, mantener los dos programas con tratamientos menos costosos, aunque fuesen menos efectivos? ¿Cómo se logra distribuir justamente los recursos escasos? ¿Cuándo debe considerarse justo o injusto un servicio de salud? ¿Cómo tomar decisiones cuando los recursos son finitos?(12).

Nos interesa reproducir los planteos que realiza Terlizzi sobre este tema: "Gracias a los debates, a las campañas de distintas organizaciones no gubernamentales, y a los esfuerzos gubernamentales y de cooperación internacional, el acceso a los medicamentos esenciales, entre ellos los del VIH/ SIDA, ha mejorado notablemente en las últimas décadas en gran parte del mundo. Aunque, claro está, aún sigue siendo un grave problema para los países con menores recursos. A diferencia de esto, el acceso a los medicamentos de alto costo (MAC) (en gran medida debido al patentamiento) es limitado y depende de las políticas de salud pública de cada país. En América Latina, solamente Venezuela, Colombia, Brasil, Uruguay, Ecuador, Cuba, Costa Rica y Argentina disponen de algún mecanismo -específico o general- de provisión de al menos ciertos MAC. Dentro de este grupo de países, algunos cuentan con programas especiales para su cobertura, como el FNR de Uruguay, mientras que otros los proveen a través del Plan Médico Obligatorio, como es el caso de Argentina. Lamentablemente, en el resto de los países de la región la provisión es escasa o inexistente. Además, el debate sobre esta clase de medicamentos es incipiente y no cuenta hasta la fecha con regulaciones especiales a nivel global. Adicionalmente, existe una fuerte presión por parte de la población -promovida en muchos casos por la industria farmacéutica- para que el estado se haga cargo de su cobertura. Por ejemplo, solamente en San Pablo, Brasil, se registraron en un solo año, el 2006, 2.927 acciones judiciales que solicitaban acceso a los MAC. En la provincia de Buenos Aires, Argentina, se registró una situación similar para el mismo período: más de 2.000 solicitudes. Aunque pareciera tratarse de un problema regional, los países europeos tampoco están exentos de las acciones judiciales para acceder a costosos tratamientos"(13).

Gracia propone los siguientes criterios en la ejecución del racionamiento de los recursos en salud : 
a) el racionamiento en virtud del principio de justicia no puede imponerlo más que quien tiene la gestión del bien común, es decir el gestor o administrador;

b) se deben gestionar eficientemente los recursos disponibles, lo que es una obligación de justicia cuando éstos son públicos;

c) es necesario evaluar la eficacia del producto que se está otorgando. Nunca hay obligación de justicia frente a procedimientos no indicados o de eficacia dudosa;

d) en la teoría de la justicia hay un principio que Rawls llama “de la diferencia”. Según él, cuando la lotería de la vida hace que ciertos bienes se repartan, la equidad exige compensar esa discriminación negativa con otra positiva, utilizando el criterio "maximum".

Manifiesta que cuando se pide a los médicos que al tomar decisiones tengan en cuenta el aspecto económico, se crea una situación de lealtades divididas; no puede ser a la vez defensor de un paciente y servir a los guardianes financieros de la sociedad(14).

Charlesworth considera que "en los años cincuenta y sesenta en las sociedades occidentales surgió la conciencia de que los recursos energéticos eran finitos y limitados, como también era finita la capacidad de la naturaleza o del ambiente para sostener el desarrollo económico y de la población". Al mismo tiempo, un reconocimiento contrario se estaba desarrollando en relación con el derecho básico de las personas a la asistencia sanitaria como una condición necesaria de la vida propiamente humana. "En el contexto general, el debate sobre la asignación de recursos se ha centrado especialmente en la evaluación económica y el racionamiento de esos recursos se hace sobre una base de costos y beneficios. Como resultado, se han tendido a abandonar las dimensiones puramente éticas del debate". "La asignación de recursos a la asistencia sanitaria no solo supone un tema de costo-beneficio-eficacia, sino que por encima de todo es un tema de justicia humana o equidad, en el que la prioridad es sopesar debidamente los intereses de cada una de las partes implicadas: pacientes, profesionales y comunidad en general"(15).

Refiriéndose a las diferentes interpretaciones sobre la justicia, expresa: "El debate ha sido dominado por un enfoque utilitarista o consecualista que determina el valor del acto médico y de las políticas sanitarias exclusivamente en términos de consecuencias medibles o resultados"... "Algunos críticos se han quejado de que la asignación racional de los recursos sanitarios no será nunca posible si los médicos no tienen en cuenta la necesidad social de racionarlos y permanezcan anclados ciegamente en las necesidades particulares del paciente que tienen delante". Ante esto, Charlesworth sostiene "en un sentido literal, el médico y los otros asistentes deben permanecer comprometidos con el uso de sus habilidades profesionales para hacer frente a las necesidades del paciente. Sin duda, el médico debe operar dentro de ciertas restricciones económicas o de cualquier otra índole, pero como médico no es asunto suyo actuar como guardián y controlar el acceso a los recursos"(15).

Hasta la aprobación en 2007 de la Ley 18.211, la obligación del Estado uruguayo consistía en brindar gratuitamente atención sanitaria solo a los indigentes y carentes de recursos suficientes. Los demás ciudadanos estaban obligados a cuidar su salud y tratarse por sus propios medios económicos. Esto producía grandes desigualdades en la atención sanitaria de la población. Esta situación cambia con la Ley 18.211 que crea el Sistema Nacional Integrado de Salud (SNIS), aprobada en diciembre de 2007. La reforma se inicia en 2008, y sigue implementándose en la actualidad, integrando en forma progresiva a los distintos grupos de la población. Exponemos el artículo 3 de dicha ley, de modo de observar los principios que la orientan(16):

Artículo $3^{\circ}$. Son principios rectores del SNIS:

A) La promoción de la salud con énfasis en los factores determinantes del entorno y los estilos de vida de la población.

B) La intersectorialidad de las políticas de salud respecto del conjunto de las políticas encaminadas a mejorar la calidad de vida de la población.

C) La cobertura universal, la accesibilidad y la sustentabilidad de los servicios de salud.

D) La equidad, continuidad y oportunidad de las prestaciones.

E) La orientación preventiva, integral y de contenido humanista.

F) La calidad integral de la atención que, de acuerdo a normas técnicas y protocolos de actuación, respete los principios de la bioética y los derechos humanos de los usuarios.

G) El respeto al derecho de los usuarios a la decisión informada sobre su situación de salud.

H) La elección informada de prestadores de servicios de salud por parte de los usuarios.

I) La participación social de trabajadores y usuarios.

J) La solidaridad en el financiamiento general.

K) La eficacia y eficiencia en términos económicos y sociales.

L) La sustentabilidad en la asignación de recursos para la atención integral de la salud.

Destacamos que nuestra normativa está orientada en los principios de la bioética y de los derechos 
humanos, como explícitamente se resalta en el inciso $\mathrm{F}$.

Este complejo tema de aplicación del principio de justicia en el ámbito de la salud muestra que existen múltiples interpretaciones y justificaciones en él, dependiendo fundamentalmente de la filosofía política que se adopte.

En el mismo artículo 3 vemos que se ha optado por la justicia como equidad y por lo tanto, a pesar de las dificultades, creemos que nuestro país se encuentra en una situación favorable en cuanto a los aspectos filosóficos.

Para evitar que se repitan situaciones derivadas del conflicto entre escasos recursos y altos costos, se debe considerar la interpretación del principio de justicia como equidad y se debe lograr consenso respecto a los criterios para la asignación de los recursos entre científicos, profesionales y, en especial, la sociedad entera.

\section{Bibliografía}

1. Gracia D. Procedimientos de decisión en ética clínica. Madrid: Eudema, 1991.

2. Jonsen AR, Siegler M, Winslade WJ. Clinical Ethics: A Practical Approach to Ethical Decisions in Clinical Medicine. 2 ed. New York: Macmillan, 1986.

3. Brussino S. Teorías éticas. Buenos Aires: Ministerio de Salud, OMS-OPS, 2015.Disponible en: http://www. salud.gob.ar/dels/entradas/teorias-bioeticas. [Consulta: 15 Febrero 2018].

4. Drane JF. Becoming a good Doctor: the place of virtue and character in medical ethics. Kansas City, MO: Sheed \& Ward, 1988.

5. Beauchamp TL, McCullough LB. Ética Médica:las responsabilidades morales de los médicos. Barcelona: Labor, 1987.

6. Organización de las Naciones Unidas para la Educación, la Ciencia y la Cultura. Declaración Universal sobre Bioética y Derechos Humanos. París: Unesco, 2005. Disponible en: http://unesdoc. unesco.org/images/0014/001461/146180S.pdf. [Consulta: 15 Febrero 2018].
7. Garrafa V. Declaración Universal de Bioética y Derechos Humanos DUBDH (ONU, 2005). Buenos Aires: Ministerio de Salud, 2017. Disponible en: http://www.salud.gob.ar/dels/entradas/declaracion-universal-sobre-bioetica-y-derechos-humanos-dubdh-onu-2005. [Consulta: 15 Febrero 2018].

8. Sass HM, Vifhues H, Mainetti JM. Protocolo de Bochum para la práctica ético médica: análisis científico y ético para el manejo de un caso médico. Bochum: Zentrum fur Medizinische Ethik Bochum, 1987.

9. Schotsmans P. La bioética en Europa. En: Cortina A. Tareas de una bioética latina. Jurisprudencia Argent 2004,4 (5 Num Esp):9-15.

10. Reyes Caorsi W, Tortajada G, Varela G. Haciendo medicina con "el diario del lunes". Rev Urug Cardiol 2018; 33(1):100-8.

11. Beauchamp TL, Childress JF. Principles of biomedical ethics. 2 ed. New York: Oxford University Press, 1983:319-20.

12. Lolas F, Outomuro D. Bioética y salud pública. En: Lolas F, Quezada A, Rodríguez, E, eds. Investigación en salud: dimensión ética. Santiago de Chile: CIEB, Universidad de Chile, 2006:103-16. Disponible en: http://web.uchile.cl/archivos/uchile/bioetica/doc/manual_bioetica\%20.pdf. [Consulta: 15 Febrero 2018].

13. Terlizzi MS. Bioética y propiedad intelectual:debates actuales en torno de las patentes sobre los Medicamentos de Alto Costo (MAC). Rev Red bioét/UNESCO 2015, 2(12):126-38. Disponible en: http://www.unesco.org/new/fileadmin/MULTIMEDIA/ FIELD/Montevideo/pdf/RevistaBioetica12.pdf. [Consulta: $15 \mathrm{Fe}-$ brero 2018 ].

14. Gracia D. Cuidados intensivos en la era de la bioética. En: Net A, ed. Ética y costes en medicina intensiva. Barcelona: Springer-Verlag Ibérica,1996:1-5.

15. Charlesworth M. La bioética en una sociedad liberal. Gran Bretaña. Cambridge: Cambridge University Press, 1996.

16. Ley 18211. Sistema Nacional Integrado de Salud: normativa referente a su creación, funcionamiento y financiación. Montevideo, 13 diciembre 2007. Disponible en: https://parlamento.gub.uy/documentosyleyes/leyes/ley/18211. [Consulta: 15 Febrero 2018]. 